\title{
Gaussian beam mode analysis of partial reflections in simple quasi-optical systems fed by horn antennas
}

\author{
J. Anthony Murphy ${ }^{\mathrm{a}, *}$, Neil Trappe ${ }^{\mathrm{a}}$, Stafford Withington ${ }^{\mathrm{b}}$ \\ ${ }^{a}$ Experimental Physics Department, National University of Ireland, Maynooth, Co. Kildare, Ireland \\ b Cavendish Laboratory, Madingley Road, Cambridge CB3 OHE, UK
}

Received 14 November 2002

\begin{abstract}
Low-level reflections are particularly troublesome in sensitive high-resolution spectral line receivers in the submillimetre and terahertz wavebands, giving rise to baseline ripple on spectra which is hard to distinguish from wide lines. In typical systems for submillimetre-wave astronomy, for example, a lens or flat dielectric plate is often used as a cryostat window placed close to a feed horn. Partial reflection from such interfaces can give rise to return loss at the horn throat, which is considerably higher than for the horn radiating into free space. A full scattering matrix approach based on a combination of standard waveguide modes and quasi-optical Gaussian beam modes can be used to analyse standing waves due to partial reflections, in which track is kept of both the backward and forward going components of the propagating fields. In this paper, we consider how such an analytical tool can be set up and we present typical examples of interest in which horn antennas feed simple components including windows, lenses and reflecting central blockages.

(c) 2003 Elsevier Science B.V. All rights reserved.
\end{abstract}

PACS: 07.57.K; 07.57.P; 95.55.J; 84.40.B

Keywords: Submillimetre-wave receivers; Quasi-optics; Standing waves

\section{Introduction}

In this paper we extend a theoretical framework based on Gaussian beam mode analysis [1] to the modeling of partial reflections and standing waves in submillimeter and terahertz quasi-optical systems fed by horn antennas. Gaussian beam modes

\footnotetext{
* Corresponding author. Tel.: +353-1-708-3771; fax: +353-1708-3313.

E-mail address: anthony.murphy@may.ie (J.A. Murphy).
}

can be thought of as the beam-guide analog of conventional waveguide modes [2], where a beam guide consists of a number of focussing elements spaced so as to ensure that the propagating beam is always quasi-collimated. Both because of truncation and aberrational effects, realistic optical components disturb the pure modal propagation, just as a step does in a waveguide. At such optical components power is scattered between modes $[3,4]$. Gaussian beam mode analysis can be applied to any quasi-optical system in which there are partial reflections by also including the backward travelling modes. Such an analysis is necessary to 
have a more realistic description of the operation and performance limitations of a complete system resulting from the presence of non-ideal optical components. To this end an analogous mode matching scattering matrix approach to that applied in wave-guide discontinuities and horn antenna modeling can be developed $[1,5]$. In such a description free space propagation of Gaussian beam modes is most conveniently represented using propagation "scattering" matrices, which are diagonal (as in waveguides of uniform crosssection) and take account of the modal phase delays.

Another level of complexity is introduced by the fact that most optical systems typical of the submillimeter and terahertz bands are coupled to horn antenna feed structures (typically single moded corrugated horn antennas). Any reflections from horn antennas do not occur at a single well-defined plane, so it is necessary to consider the questions about "how far" into the horn an incoming field propagates before being reflected. This can be tackled by combining an electromagnetic approach based modal analysis of the feed structure with a beam guide modal analysis of the optical system, as will be discussed in Section 2. In Section 3 , we consider specific examples of partial reflections from transparent windows and lenses (of finite thickness), as well as at reflecting blockages of the beam (such as occurs in the center of the field for an on-axis Cassegrain antenna system, for example). All of these components give rise to low level standing wave effects, which are a particular nuisance for heterodyne systems.

\section{Theory}

The modal matching technique can be regarded as a method for obtaining the overall transmission and reflection properties of any guide structure in which modes of propagation can be defined. In our case the guide structure consists of a horn feeding a quasi-optical system. Any section of the system (or the system as a whole) is represented by a single scattering matrix $[S]$ with the reflection and transmission characteristics determined by the equation:
$\left[\begin{array}{l}{[B]} \\ {[D]}\end{array}\right]=\left[\begin{array}{ll}{\left[S_{11}\right]} & {\left[S_{12}\right]} \\ {\left[S_{21}\right]} & {\left[S_{22}\right]}\end{array}\right]\left[\begin{array}{l}{[A]} \\ {[C]}\end{array}\right]$.

$[A]$ and $[B]$ are vectors containing the forward and reflected mode coefficients, $A_{n}$ and $B_{n}$, respectively, at the input side. $[C]$ and $[D]$ are vectors of the mode coefficients, $C_{n}$ and $D_{n}$, of all the modes at the output plane. Clearly, the modes at the output plane are not the same (in terms of scale size) as the modes at the input plane. They also may not be of the same form (i.e in the sense of whether free space or waveguide).

\subsection{Non-scattering beam guide propagation}

In a cylindrical beam guide coupled to a conical horn (smooth walled or corrugated) it is most appropriate to use Associated Laguerre Gaussian modes, which for a wave travelling in the positive $z$-direction (away from a waist at $z=0$ ) have a mathematical form given by

$$
\begin{aligned}
\left\{\begin{array}{c}
\Psi_{n}^{\alpha, \cos }(r, \phi, z) \\
\Psi_{n}^{\alpha, \sin }(r, \phi, z)
\end{array}\right\}= & \Psi_{n}^{\alpha}(r, z)\left\{\begin{array}{c}
\cos (\alpha \phi) \\
\sin (\alpha \phi)
\end{array}\right\} \\
= & \sqrt{\frac{2\left(2-\delta_{0 n}\right) n !}{\pi W^{2}(n+\alpha) !}\left(2 \frac{r^{2}}{W^{2}}\right)^{\alpha / 2}} \\
& \times L_{n}^{\alpha}\left(2 \frac{r^{2}}{W^{2}}\right) \exp \left(-\frac{r^{2}}{W^{2}}\right) \\
& \times \exp \left(-\mathrm{i} k\left[z+\frac{r^{2}}{2 R}\right]\right. \\
& \left.+\mathrm{i}(2 n+l+1) \arctan \left[\frac{\pi W^{2}}{\lambda R}\right]\right) \\
& \times\left\{\begin{array}{l}
\cos (\alpha \phi) \\
\sin (\alpha \phi)
\end{array}\right\} .
\end{aligned}
$$

$\alpha$ is an integer representing the degree of the Laguerre polynomial. $W$ and $R$ are the beam width parameter and phase radius of curvature, respectively, and $\Delta \phi_{00}=\arctan \left(\pi W^{2} / \lambda R\right)$ is the phase slippage for the fundamental mode between the waist and the plane of interest (see, e.g., [2]). A mode travelling "backwards" in the negative $z$ direction, $\Psi_{n}^{\alpha,(-)}$ can be obtained by choosing the appropriate monochromatic modal solution for the Helmholtz equation, and is equivalent to setting $z \rightarrow-z$. This changes the sign of $k z$ and $R$, but 
not $W$. The appropriate sign for the phase slippage also changes in (2), if it is assumed that the phase slippage is zero at the origin of $z$. Therefore, the relationship between a forward $\Psi_{n}^{\alpha,(+)}$ and a backward travelling mode with the same propagation characteristics (i.e. waist at the same position and of the same size) can be expressed as $\Psi_{n}^{\alpha,(-)}=$ $\left[\Psi_{n}^{\alpha,(+)}\right]^{*}$. It is assumed that for both sets of solutions the time dependence factor is included in the same $\exp (+\mathrm{i} \omega t)$ factor.

The phase evolution associated with lossless propagation in a quasi-optical system is conveniently incorporated into a propagation matrix $[P]$, which operates on the mode amplitudes (as in the case of a true scattering matrix) such that, $\left[P_{11}\right]=\left[P_{22}\right]=[0]$ and $\left[P_{12}\right]=\left[P_{21}\right]=[V]$. This is the same as for waveguide modes in a uniform waveguide [5]. Here $[V]$ represents a diagonal matrix of on-axis phase evolution terms of the form $V_{v v}=\exp \left(\mathrm{i} \Delta \Phi_{n \alpha}\right)$, where for Laguerre modes $v$ represents a mode of order $n$ and degree $\alpha$. For modes travelling in a uniform beam guide of length $d$ the relevant phase term is: $\Delta \Phi_{n \alpha}=-k d+$ $(2 n+\alpha+1) \Delta \phi_{00}$. The on-axis phase slippage of the fundamental mode with respect to a plane wave, $\Delta \phi_{00}$, depends on the input beam parameters $\left(R_{\text {in }}\right.$ and $\left.W_{\text {in }}\right)$, as well as the quasi-optical beam guide configuration (most conveniently expressed in terms of an $A B C D$ matrix) [6,7], and is given by: $\Delta \phi_{00}=-\operatorname{Arg}\left(A+B / q_{\text {in }}\right)$, where $1 / q_{\text {in }}=1 / R_{\text {in }}-$ $j \lambda / \pi W_{\text {in }}^{2}$, with the subscript "in" implying parameters at the input plane. The submatrix $[V]$ transforms the vector of input amplitudes $A_{v}$ and $C_{v}$ into a vector of amplitudes $D_{v}$ and $B_{v}$ so that $D_{v}=V_{v v} A_{v}$ and $B_{v}=V_{v v} C_{v}$.

\subsection{Propagation in dielectrics and reflections from dielectric interfaces}

In a non-scattering beam guide the assumption is made that all lenses are perfect phase transformers. If one is interested in dealing with real lenses, but can still assume paraxial quasicollimated propagation, then two additional physical effects have to be adequately accounted for, these being partial reflection at the dieletric interfaces and propagation in the finite thickness of the dielectric material.
As a Gaussian beam mode enters a dielectric medium there is a sudden change in the wavelength from $\lambda_{\mathrm{o}}$ to $\lambda_{\mathrm{n}}=\lambda_{\mathrm{o}} / n$ in the medium. This results in a change in the phase curvature for the beam from $R_{0}$ in free space to $R_{\mathrm{n}}=n R_{0}$ in the medium (assuming a flat interface), where $n$ is the refractive index of the medium. It turns out to be convenient for propagation inside a dielectric to introduce a reduced complex phase radius of curvature $q^{\prime}=q / n$, on which the $A B C D$ matrix operates. This is convenient as $q^{\prime}$ does not change for the beam across a flat transverse dielectric interface, since

$\frac{1}{q_{\mathrm{n}}^{\prime}}=\frac{n}{R_{\mathrm{n}}}-\mathrm{i} \frac{n \lambda_{\mathrm{n}}}{\pi W}=\frac{1}{R_{0}}-\mathrm{i} \frac{\lambda_{0}}{\pi W}=\frac{1}{q_{\mathrm{o}}^{\prime}}$,

where subscript ' $n$ ' refers to inside the medium at the interface and ' $o$ ' refers to outside the medium in free space. This follows the convention of Siegman [7], in which he uses reduced radius of curvature $R^{\prime}=R / n$.

In the general case we require the same type of relationship for the reduced complex phase radii of curvatures $q_{\text {in }}^{\prime}$ and $q_{\text {out }}^{\prime}$ between input and output planes as in free space, i.e.

$q_{\mathrm{out}}^{\prime}=\frac{A q_{\mathrm{in}}^{\prime}+B}{C q_{\mathrm{in}}^{\prime}+D}$.

The corresponding appropriate $A B C D$ matrix for propagation in a dielectric medium is $\{1, d / n, 0,1\}$. For a curved interface between two media of refractive indices $n_{1}$ and $n_{2}$ the appropriate matrix is given by $\left\{1,0,\left(n_{2}-n_{1}\right) / R_{\text {surf }}, 1\right\}$, where subscripts 1 and 2 refer to incident and transmission regions, respectively, and $R_{\text {surf }}$ is the radius of curvature of the interface with the usual convention for $R$ positive. In the generalised case the phase slippage is given by

$\Delta \phi_{n \alpha}=(2 n+\alpha+1) \operatorname{Arg}\left(-A-\frac{B}{q_{\mathrm{in}}^{\prime}}\right)$.

For paraxial beams we can approximately assume that, for each point on the interface, the reflected and transmitted electric fields are given by the Fresnel equation for normal incidence: $E_{\text {refl }}=$ $\rho E_{\text {inc }}$, where $\rho=\left(n_{1}-n_{2}\right) /\left(n_{1}+n_{2}\right)$, and $E_{\text {trans }}=$ $\tau E_{\text {inc }}$, with $\tau=2 n_{1} /\left(n_{1}+n_{2}\right)$. For the transmitted field this simply implies for the forward going 
wave that $\left[S_{21}\right]=\tau\left[\delta_{\mu v}\right]$. The case for the reflected field is more complicated since the phase front curvature is reversed on reflection, so modes are scattered. $E_{\text {refl }}$ can be written in terms of the modes travelling in the backward direction $E_{\text {refl }}=$ $\sum_{i} B_{\mu} \psi_{\mu}^{(-)}$, which have their waists coinciding with those for the forward going waves. Writing $E_{\text {inc }}$ as a sum of modes travelling in the forward direction $E_{\text {inc }}=\sum_{i} A_{\mu} \psi_{\mu}^{(+)}$, implies the $B_{\mu}$ can be derived from the scattering relationship: $B_{\mu}=\sum_{j}\left[S_{11}\right]_{\mu v} A_{v}$, where

$\left[S_{11}\right]_{\mu v}=\rho \int_{A}\left\{\psi_{v}^{(-)}\right\}^{*} \psi_{\mu}^{(+)} r \mathrm{~d} r \mathrm{~d} \phi$,

and $A$ represents the interface surface over which integration takes place. With respect to integration over the tangential plane, the typically spherical interface introduces extra spherical phase error terms of $\exp ( \pm \mathrm{i} \delta(r))$. For the forward travelling wave $\delta(r)=+\pi n r^{2} / \lambda_{\mathrm{o}} R_{\text {surf }}$, with $R_{\text {surf }}$ being the radius of curvature of the surface and $n$ the refractive index of the medium in which the reflection occurs. Thus,

$\left[S_{11}\right]_{\mu v}=\rho \int_{\mathrm{TP}}\left\{\psi_{v}^{(-)}\right\}^{*} \exp (\mathrm{i} 2 \delta(r)) \psi_{\mu}^{(+)} r \mathrm{~d} r \mathrm{~d} \phi$,

where now the integration is over the tangent plane and the phase errors for both waves are included.

\subsection{Transformation of waveguide modes to free space modes}

At the horn aperture the forward going waveguide modes are launched into free space while the backward propagating waveguide modes can be coupled to the horn antenna. We therefore need to consider how to combine the horn and beam guide mode matching techniques by deriving the appropriate transformation matrices. The transverse electric field $\mathbf{e}_{\text {total }}$ at the mouth of the horn can be written as a linear sum of TE and TM modal fields. If one is considering conical horns (both smooth walled and corrugated) the waveguide segments are cylindrical. For such cylindrical waveguides of radius $a$, the transverse electric fields of the corresponding TE and TM modes are

$$
\begin{aligned}
\mathbf{e}_{l}^{\mathrm{TE}}= & \frac{J_{0}\left(p_{1 l}^{\prime} r / a\right) \mathbf{i}+J_{2}\left(p_{1 l}^{\prime} r / a\right)[\cos 2 \phi \mathbf{i}+\sin 2 \phi \mathbf{j}]}{\sqrt{2 \pi a^{2}\left(J_{1}\left(p_{1 l}^{\prime} r / a\right)^{2}-J_{2}\left(p_{1 l}^{\prime} r / a\right)^{2}\right)}}, \\
\mathbf{e}_{l}^{\mathrm{TM}} & =\frac{J_{0}\left(p_{1 l} r / a\right) \mathbf{i}-J_{2}\left(p_{1 l} r / a\right)[\cos 2 \phi \mathbf{i}+\sin 2 \phi \mathbf{j}]}{\sqrt{2 \pi a^{2}\left(J_{2}\left(p_{1 l} r / a\right)^{2}\right)}},
\end{aligned}
$$

where $p_{1 l}$ represents the $l$ th zero of $J_{1}(z)$, and $p_{1 l}^{\prime}$ represents the $l$ th zero of $J_{1}^{\prime}(z)$. The constant of proportionality has been chosen for convenience to make $\int_{A}\left|\mathbf{e}_{l}^{\mathrm{TE} / \mathrm{TM}}\right|^{2} r \mathrm{~d} r \mathrm{~d} \phi$ equal to unity. On ordering the guide modes by their cutoff wavelength, so that those of odd order are TE while those of even order are TM we obtain

$\mathbf{e}_{\text {total }}=\sum_{l} \alpha_{l} \mathbf{e}_{l}^{\mathrm{TE}}+\beta_{l} \mathbf{e}_{l}^{\mathrm{TM}}=\sum_{n} A_{n} \mathbf{e}_{n}^{\mathrm{G}}$,

where $A_{2 n-1}=\alpha_{n}, A_{2 n}=\beta_{n}, \mathbf{e}_{2 n-1}^{\mathrm{G}}=\mathbf{e}_{n}^{\mathrm{TE}}$ and $\mathbf{e}_{2 n}^{\mathrm{G}}=$ $\mathbf{e}_{n}^{\mathrm{TM}}$.

At the interface between the horn and "free space" the description of the field emerging from the horn must be transformed into a sum involving free space modes. Because of symmetry the waveguide modes of a conical horn will clearly couple only to free space Associated Laguerre Gaussian modes for which $\alpha=0$ or 2. Thus, we can expand each waveguide mode as

$\mathbf{e}_{v}^{\mathrm{G}}=\sum_{n} T_{\mu \nu}^{0} \psi_{\mu}^{0} \mathbf{i}+T_{\mu \nu}^{2} \psi_{\mu}^{2}[\cos 2 \phi \mathbf{i}+\sin 2 \phi \mathbf{j}]$.

Here $T_{\mu \nu}^{0}=\int_{A}\left(\psi_{\mu}^{0} \mathbf{i}\right)^{*} \cdot \mathbf{e}_{v}^{\mathrm{G}} r \mathrm{~d} r \mathrm{~d} \phi$, and $T_{\mu \nu}^{2}=\int_{A}\left(\psi_{\mu}^{2} \mathbf{n}\right)^{*}$. $\mathbf{e}_{v}^{\mathrm{G}} r \mathrm{~d} r \mathrm{~d} \phi$, where $\mathbf{n}$ represents the unit vector $\mathbf{n}=\cos 2 \phi \mathbf{i}+\sin 2 \phi \mathbf{j}$. Thus, at the horn aperture we can re-express the overall field $\mathbf{e}_{\text {total }}=\sum_{v} A_{v} \mathbf{e}_{v}^{\mathrm{G}}$ in terms of Associated Laguerre Gaussian modes

$\mathbf{e}_{\text {total }}=\sum_{\mu} D_{\mu}^{0} \psi_{\mu}^{0} \mathbf{i}+D_{\mu}^{2} \psi_{\mu}^{2}[\cos 2 \phi \mathbf{i}+\sin 2 \phi \mathbf{j}]$,

where now clearly, $D_{\mu}^{0}=\sum_{m} T_{\mu v}^{0} A_{v}$ and $D_{\mu}^{2}=$ $\sum_{n} T_{\mu v}^{2} A_{v}$. The transformation matrix [T] can be regarded as the $\left[S_{21}\right]$ submatrix component of a scattering matrix, which transforms the mode amplitudes associated with waveguide modes into those associated with beam guide free space modes. Clearly, in this case $\left[S_{12}\right]=\left[S_{21}\right]^{\mathrm{T}}$, where superscript ' $T$ ' represents transpose. Note that for a horn whose aperture is several wavelengths wide 
one can assume that there is no appreciable reflection of the waveguide modes at the aperture of the horn itself. This is because only the highest order modes, which contribute little to the beam, will have a guide impedance appreciably different from that of free space. This implies that $\left[S_{11}\right]=$ [0]. Similarly, if we assume that any free space radiation propagating towards the horn, and which misses the aperture, is absorbed (or at least not reflected back), then $\left[S_{22}\right]=[0]$. The part of the cross section of the quasi-optical beam that is intercepted by the horn will be coupled to waveguide modes. If we take the effects of a flat transverse reflecting horn flange into account then clearly $\left[S_{22}\right]$ needs to be calculated.

\section{Examples}

\subsection{Dielectric windows with corrugated horn feeds}

All the elements necessary to analyse some simple quasi-optical configurations are now in place. We first consider applying the above theory to an example case of a corrugated conical horn antenna which looks through a thin partially reflecting dieletric sheet placed close to the horn aperture (see Fig. 1). This is a set-up that occurs in practice when detection systems have to be cryogenically cooled and any incident radiation is coupled to the horn through a cryostat window. We assume in this example that the plane of the sheet is perpendicular to the axis of the horn and that there are no matching layers. The partially reflecting sheet is also assumed to be of uniform thickness, at least to within a small fraction of a wavelength. In the example considered, the wave- length of the radiation is taken to be $1 \mathrm{~mm}$, the horn aperture has a diameter of $4 \lambda$ and the axial length of the horn is $40 \lambda$. The dieletric sheet $(n=2.00)$ is placed close to the horn at a distance of $5 \lambda$, in front of the horn. The horn beam has a focal ratio of F3.5, implying the beam has a waist radius of $W_{0}=\lambda / \pi \theta_{0}$, where $\theta_{0}$ is the semi-opening angle to the $1 / e$ point in amplitude (so that $\left(2 \theta_{0}\right)^{-1} \approx 3.5$ ).

To get a feeling for the level of reflections that might be a nuisance and contribute to standing waves we consider the reflection submatrix $\left[S_{11}^{\text {win }}\right]_{00}$ term for just the window itself as a function of its thickness (Fig. 1a). This represents the amount of power carried by the fundamental Gaussian that is reflected back in the direction of the horn and therefore can couple well to the horn. It is assumed here that the Gaussian beam mode set is chosen to maximise the power carried by the fundamental in the description of the horn beam.

For an accurate calculation of course we use the full $\left[S_{11}^{\text {total }}\right]_{00}$ matrix, including the horn plus the quasi-optical system it feeds, to compute the total amount of power reflected back at the horn throat into the waveguide (Fig. 1b). Fig. 2a shows the power reflection coefficient for the $\mathrm{TE}_{11}$ mode (fraction of power reflected back along the guide) as a function of the thickness of the absorber, expressed as a fraction of the wavelength in the medium. (Note horn feeds a circular waveguide with a transition section which produces a free space return loss of approximately $-30 \mathrm{~dB}$.) Also shown is the reflected power at the window carried by the fundamental Gaussian $\left|\left[S_{11}^{\text {win }}\right]_{00}\right|^{2}$.

We see the reflected power in the fundamental Gaussian for the window is a good approximation to the amount of power actually reflected at the
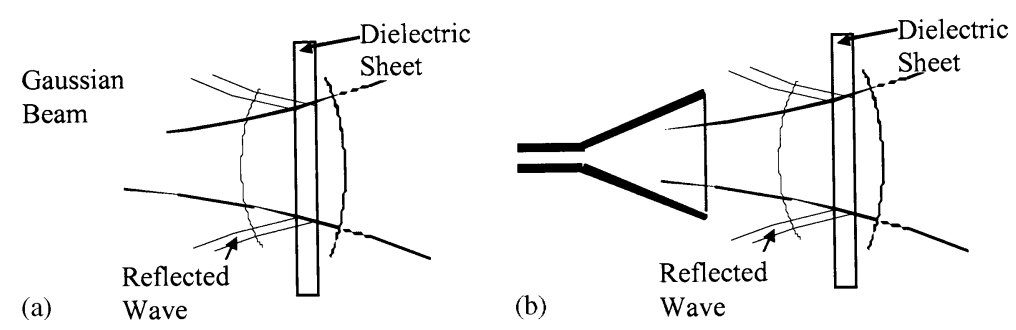

Fig. 1. (a) Gaussian beam and (b) horn antenna (corrugated conical) illuminating a partially reflecting dielectric sheet. 

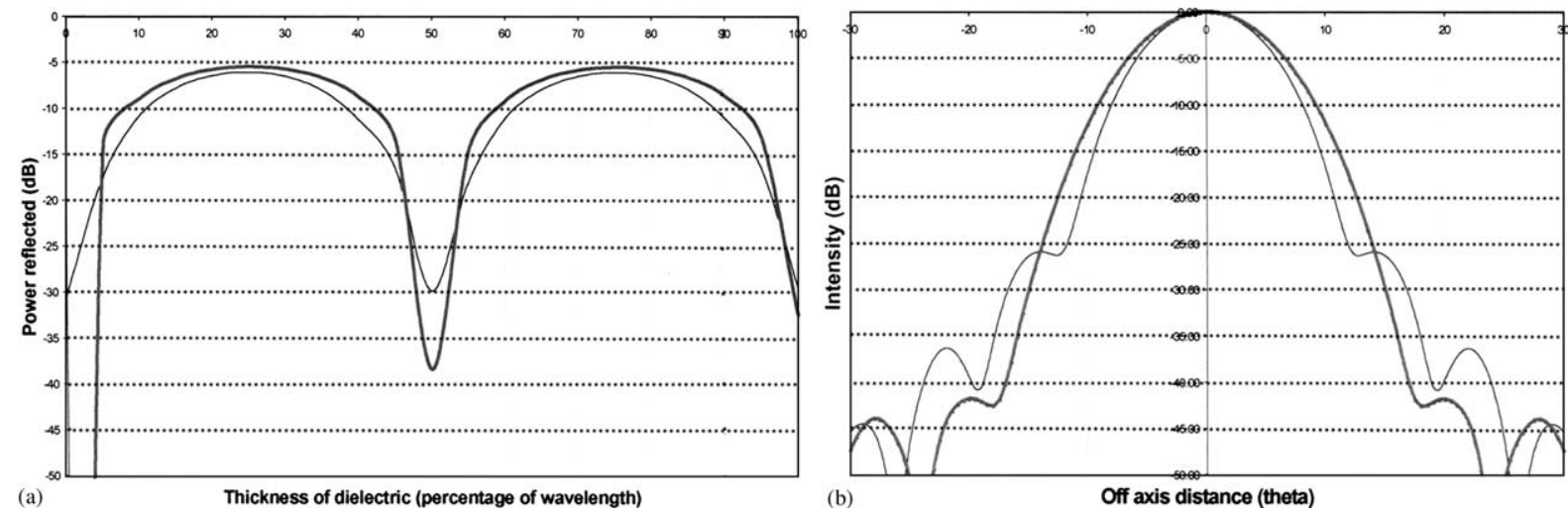

Fig. 2. (a) Fraction of power $R$ reflected in the fundamental Gaussian $\left|\left[S_{11}^{\text {win }}\right]_{00}\right|^{2}$ (bold line) and the reflection coefficient at waveguide throat $\left|\left[S_{11}^{\text {total }}\right]_{00}\right|^{2}$ for the horn as function of the thickness of the dielectric window in terms of wavelength in dielectric (thin line) and (b) beam pattern for horn antenna for cases where reflection from dielectric is maximum (bold line) and minimum (thin line).

horn throat. This is because a corrugated horn couples well to a Gaussian beam of the appropriate width. Of course, when the thickness of the dielectric is zero, the $\left[S_{11}^{\text {win }}\right]_{00}$ term for the window is zero, whereas the reflection coefficient for the real horn remains finite due to internal reflections within the waveguide structure. This information can be vital for understanding the effect of reflections on the performance of any component in the waveguide; for example, a mixer diode.

Fig. 2b compares the resulting beam patterns of the horn for the cases where the total power reflected from the dielectric sheet is a maximum and a minimum. Radiation patterns significantly different in width are observed, which are sensitively dependent on the dielectric thickness used. In the case with resonant dielectric thickness, the farfield pattern of the horn remains unaffected. It is also noteworthy that when the thickness of the dielectric is such as to cause maximum reflection the farfield pattern becomes highly Gaussian in shape. This can be understood in terms of the higher order modes that are reflected losing power past the horn aperture and illustrates the importance of using resonant cryostat windows.

\subsection{Lenses with corrugated horn feeds}

For a lens both the thickness of the lens and the curvature of the faces influence the standing wave effects. Typically in a beam-guide the system of lenses is used to re-collimate the beam so at least one the faces of the lens will usually be convex. As a simple example to probe the scattering technique presented above we consider the cases of a planoconvex lens used to recollimate the beam from a horn. We consider two cases: (a) the convex face is oriented towards the incident radiation so that the reflected field tends to scatter into wide angles and not couples well back into the horn, and (b) the plane face is oriented towards the horn and the convex face refocuses the reflected radiation so it can couple more efficiently to the horn, as illustrated in Fig. 3.

We take the same horn geometry as from previous examples with a horn feeding a lens at a distance of $30 \lambda$ from the horn aperture. The lens has a focal length of $30 \lambda$ and a refractive index $n$ of 2.00. The lens diameter was set to $5 W$, where $W$ is the beam radius at the lens face, and the finite thickness of the lens is varied over one wavelength of the radiation in the lens medium $\left(\lambda_{\mathrm{o}} / n\right)$. A plot of the reflection coefficient at the throat of the horn $\left|\left[S_{11}^{\text {total }}\right]_{00}\right|^{2}$ versus thickness of the lens is shown in Fig. $4 \mathrm{a}$ and $\mathrm{b}$ below, for the cases where the lens has its curved face towards and away from the horn, respectively. As expected the reflection coefficient at the throat of the horn varies with the thickness of the lens as a function of $\lambda_{n}$. Since the front and back faces of the lens are not both flat, 

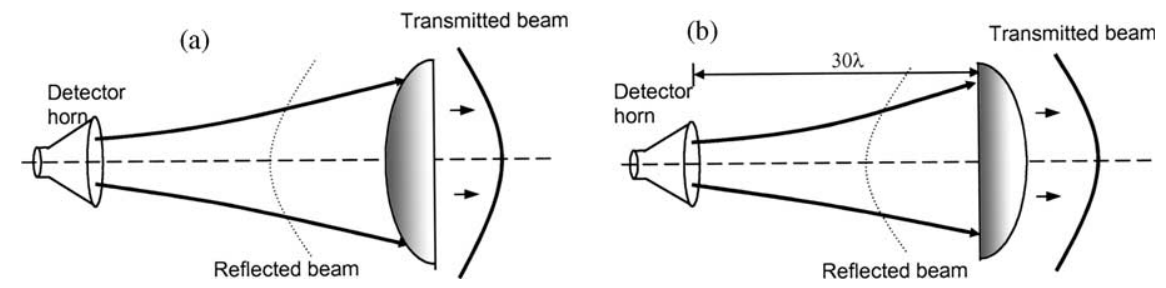

Fig. 3. The lens configurations in front of the detector horn: (a) curved face towards horn and (b) flat face towards horn. Configuration (b) should focus more reflected power towards the horn aperture.
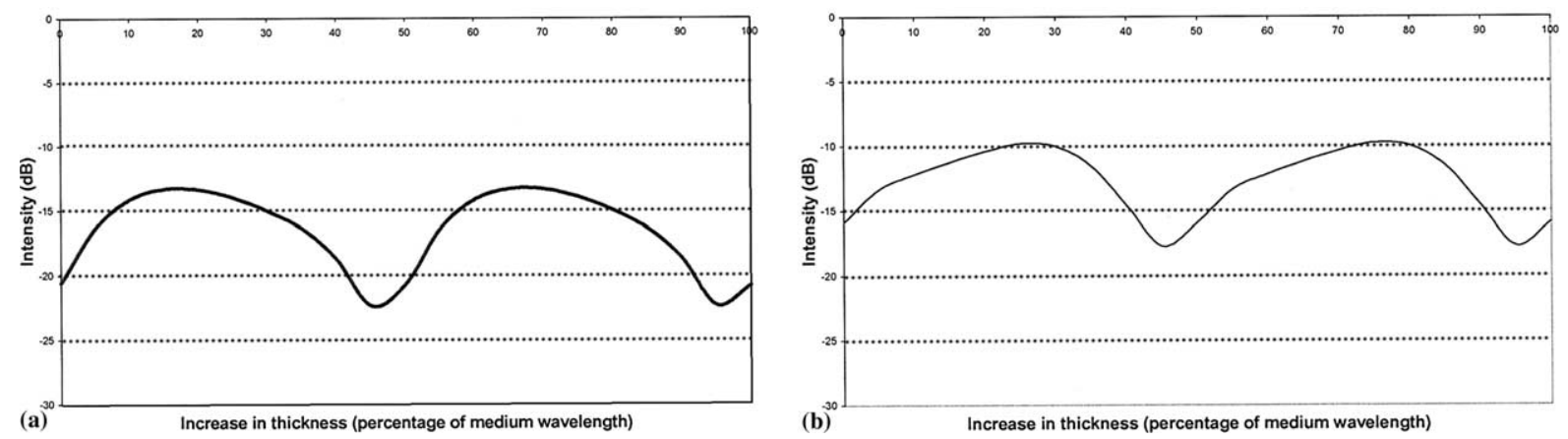

Fig. 4. The reflection coefficients at the throat of the corrugated horn as a function of the lens thickness (in terms of medium wavelength): (a) shows the reflection coefficient when the lens is in the configuration of Fig. 3a and (b) and when the lens is in the configuration of Fig. 3b. A larger reflection is seen in (b), as the lens tends to focus more energy towards the horn.

the elimination through complete destructive interference of the reflected waves cannot be achieved in this case.

The phase slippage between the modes as they propagate leads to the slight shifting of the reflection coefficient minima from exact multiples of $\lambda_{n} / 2$. The variation of reflected power with lens width is similar for both cases. As the incident radiation is dispersed at the first face of the lens in configuration (a), less power is directed towards the horn aperture, as expected. In configuration (b) the curved face of the lens focuses reflected power back towards the horn and the reflection coefficient is greater as expected ( $5 \mathrm{~dB}$ higher).

\subsection{Reflections due to central blockages}

When a quasi-optical system feeds a Cassegrain telescope a low level standing wave can be set up between the detector feed horn and the secondary mirror of the telescope. This process can be regarded as the detector horn being able to observe an image of itself in the secondary mirror. The standing wave resulting may give rise to unwanted frequency sensitivity in either receiving or transmitting systems. The telescope configuration used in the analysis is shown in Fig. 5 in which an equivalent lens represents the quasi-optical system. For this example, an image of the detector horn aperture is assumed to be produced at the secondary mirror (for optimized aperture efficiency

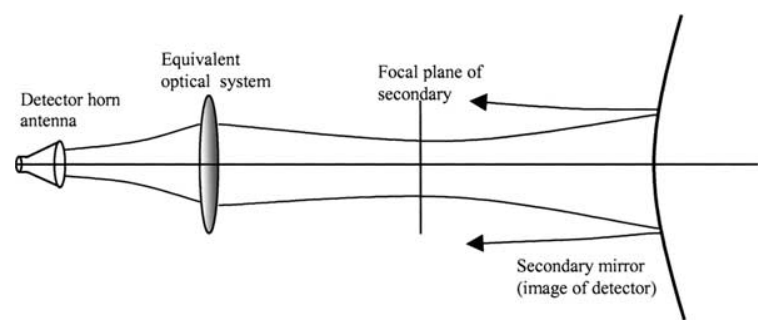

Fig. 5. The equivalent optical system used to model standing waves that can exist between the feed horn and the secondary mirror of a Cassegrain receiver. 
with a single pixel telescope, see, e.g., [8]). The horn aperture field is propagated to the Cassegrain focal plane of the telescope where a waist is formed. It is then assumed that the secondary mirror is in the far field of this waist.

A wavelength of $1 \mathrm{~mm}$ was again assumed in the calculation with the same corrugated detector horn dimensions as in previous examples. The optical system produces a beam waist at the Cassegrain focal plane with a magnification factor of 3 . The beam waist at the focal plane is then $3 W_{\mathrm{oh}}$, where $W_{\text {oh }}$ is the Gaussian beam radius of the beam at the horn. The distance from the Cassegrain focal plane to the secondary mirror is set to $2.5 \mathrm{~m}$, a typical distance for large quasi-optical telescopes. The equivalent optical system is assumed perfect with no truncation or aberrations introduced to the beam. The diameter of the secondary mirror is set to be $W_{\text {sec }} / 0.89$ (where $W_{\text {sec }}$ is the Gaussian beam radius of the beam at the secondary) to achieve optimum coupling to a distant source in the sky (optimised aperture efficiency).

Fig. 6 shows the reflection coefficient for the $\mathrm{TE}_{11}$ mode (fraction of power reflected back along the guide) as a function of $\Delta z$, an extra distance propagated between the Cassegrain focal plane and the secondary mirror. This extra distance in effect models small displacements (of the order of a few wavelengths) of the secondary mirror relative to the rest of the optical system. As can be seen the

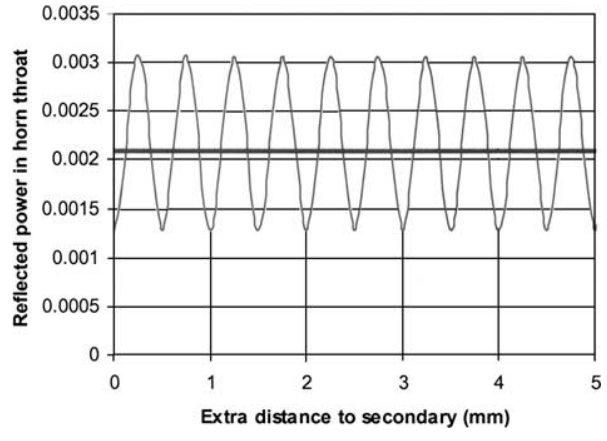

Fig. 6. Reflected $\mathrm{TE}_{11}$ mode in the throat of horn feeding Cassegrain system as the distance between the horn and the secondary mirror is varied in terms of wavelength. The constant return loss (bold line) for the horn is also shown.

reflected power in the waveguide of the horn feeding the system is a function of the extra distance travelled to the secondary mirror with minima occurring at half integral values of wavelength. The maximum reflected power is of the order of $0.3 \%$ for the specific example given. The reflected power in the horn waveguide is never zero because of the internal reflection internally within the horn.

In a second example involving a Cassegrain system, it is also possible to observe standing wave effects over a finite bandwidth by varying the wavelength at which the analysis is undertaken including the bandwidth effects on the feed horn.

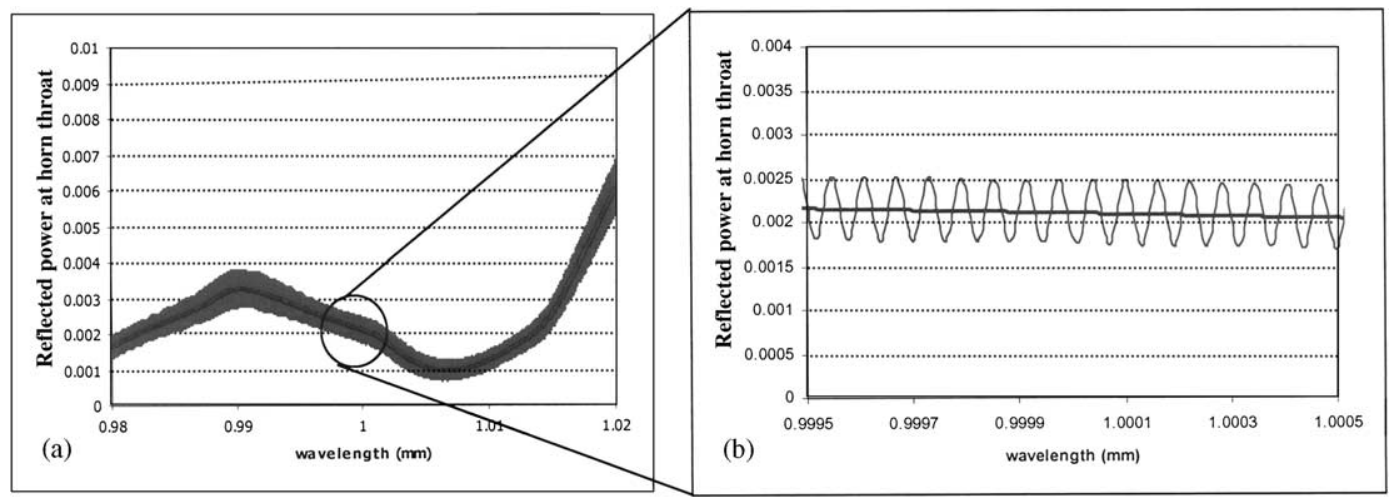

Fig. 7. (a) The standing wave observed as the wavelength of the radiation is varied between the feed horn and the secondary mirror in a typical Cassegrain receiver set-up. The constant return loss due to internal reflections within the horn is also shown (bold line) and the standing wave is seen to oscillate about this reflection coefficient. (b) Smaller bandwidth section showing standing wave oscillating about reflection coefficient. 
An image of the horn aperture is assumed to be produced with a wavelength independent optical system at the secondary mirror located $4 \mathrm{~m}$ from the horn. As the wavelength is varied (around the design wavelength of $1 \mathrm{~mm}$ ) Fig. 7a shows a plot of the reflection coefficient for the $\mathrm{TE}_{11}$ mode at the throat of the feed horn, along with the return loss, due to internal waveguide mode reflections. The return loss for the horn itself does not remain constant over a finite bandwidth due to the wavelength dependence on the corrugation depths. The standing wave present between the secondary mirror and the feed horn is seen to oscillate about the horn return loss, seen in more detail over a smaller bandwidth in Fig. $7 b$.

\section{Conclusions}

In this paper, we have presented a powerful approach based on the mode matching technique which allows for the full scattering matrix analysis of quasi-optical systems fed by horn antennas. The examples considered were of a conical corrugated horn illuminating a thin dielectric sheet, a lens and a Cassegrain telescope. The same method can be easily applied to more complicated optical systems and to other types of horns, such as smooth walled conical and Potter horns.

Some approximations are inherent in the approach, especially with regard to the paraxial nature of the quasi-optical propagation. Furthermore it is assumed that there is a smooth transition in impedance to free space at the horn aperture. Further development of the techniques could address some of these issues and include the effects of the small reflections at the horn aperture, for example, as well as the effects due to the flange surrounding the aperture. The present approach can also be extended to low loss and potentially high $Q$ systems such as two horns coupled well together in which any mismatches will give rise to strong standing waves in the system.

\section{Acknowledgements}

The authors wish to acknowledge the financial assistance of Enterprise Ireland, for partially funding this research. Neil Trappe is appreciative of the support of NUIM through a Daniel O'Connell Scholarship.

\section{References}

[1] R. Padman, J.A. Murphy, A scattering matrix formulation for Gaussian beam-mode analysis, in: Proceedings of ICAP, York, April 1991.

[2] P. Goldsmith, Quasioptical Systems: Gaussian Beam Quasioptical Propagation and Applications, IEEE Press, New York, 1998, pp. 26-32.

[3] J.A. Murphy, S. Withington, A. Egan, Mode conversion at diffracting apertures in millimetre and submillimeter-wave optical systems, IEEE Trans. Microwave Theory Technol. 41 (October) (1993) 1700-1702.

[4] S. Withington, J.A. Murphy, K.G. Isaak, Representation of mirrors in beam waveguides as inclined phase-transforming surfaces, Infrared Phys. Technol. 36 (March) (1995) 723734.

[5] A.D. Olver, P.J.B. Clarricoats, A.A. Kishk, L. Shafai, Microwave Horns and Feeds, IEEE Press, New York, 1994, pp. 100-117.

[6] D.H. Martin, J.W. Bowen, Long wavelength optics, IEEE Trans. MTT 41 (1993) 1676-1690.

[7] A.E. Siegman, Lasers, University Science Books, Mill Valley, 1986.

[8] R. Padman, J.A. Murphy, R.E. Hills, Gaussian mode analysis of Cassegrain antenna efficiency, IEEE Trans. Antennas Propagat. 35 (1987) 1093-1103. 